\title{
Pelvic malakoplakia presenting as endometrial cancer: a case report
}

\author{
Jeong Soo Cho, MD, Hye In Kim, MD, Jung Yun Lee, MD, Eun Ji Nam, MD, Sunghoon Kim, MD, \\ Young Tae Kim, MD, Sang Wun Kim, MD, PhD \\ Department of Obstetrics and Gynecology, Institute of Women's Life Medical Science, Yonsei University College of Medicine, Seoul, Korea
}

Malakoplakia is a rare granulomatous, inflammatory disease generally manifesting as ulcers of the urogenital tract, especially in the bladder, but it can occur in any part of the body. Because of its varied clinical presentations, malakoplakia is considered for differential diagnosis upon suspicion. The final diagnosis is confirmed by the presence of Michaelis-Gutmann bodies. We report a case of pelvic malakoplakia accompanied by left lower quadrant pain that was misdiagnosed as endometrial cancer with pelvic mass based on imaging studies. The patient underwent dilatation and curettage, and the pathology report revealed no malignancy. Because of persistent pain and septic shock, she underwent a debulking operation to remove the mass. Histopathologic examination revealed malakoplakia. For postoperative management, she received broad-spectrum antibiotics, but abdominal pelvic computerized tomography performed on postoperative day 9 revealed pelvic mass recurrence. To the best of our knowledge, this is the only rare case report of pelvic malakoplakia mimicking endometrial cancer.

Keywords: Malakoplakia; Endometrial cancer; Pelvic inflammatory disease; Menopause

\section{Introduction}

Malakoplakia was first announced by Michaelis and Gutmann in 1902 and named the lesion malakoplakia from Greek malakos (soft), and plaka (plaque) by Vom Hansemann in 1903. Malakoplakia is a rare non-malignant inflammatory granulomatous disease that most commonly affects the genitourinary tract, but the involvement of other organs has also been reported [1]. Even though the pathogenesis is not completely known, a number of cases accompany bacterial infection in the pelvic and as a result, is diagnosed as tuboovarian abscess (TOA) at first. The theories about etiology include bacterial infection and abnormal immune response such as abnormal function of macrophage. The immunologic theory also explains the fact that the most commonly affected patients are the immune-suppressed ones $[2,3]$.

The treatment of malakoplakia includes medical treatment such as antibiotics, surgical treatment, and sometimes withdrawal of immunosuppressive therapy [3]. Prognosis depends on not only the severity, location and extent of the disease but also the general health of the patients before the diagnosis.
We present a case which was first suspected as a severe infection involving left ovary and salpinx and as endometrial cancer later. After coming to our hospital, debulking surgery was done and the pathologist diagnosed her as malakoplakia. With conservative management including proper antibiotics after the surgery, she was fully recovered and discharged without her chronic pelvic pain.

Received: 2019.12.20. Revised: 2020.01.29. Accepted: 2020.03.19. Corresponding author: Sang Wun Kim, MD, PhD

Department of Obstetrics and Gynecology, Institute of Women's Life Medical Science, Yonsei University College of Medicine, 50-1 Yonsei-ro, Seodaemun-gu, Seoul 03722, Korea

E-mail: san1@yuhs.ac

https://orcid.org/0000-0002-8342-8701

Articles published in Obstet Gynecol Sci are open-access, distributed under the terms of the Creative Commons Attribution Non-Commercial License (http://creativecommons. org/licenses/by-nc/3.0/) which permits unrestricted non-commercial use, distribution, and reproduction in any medium, provided the original work is properly cited.

Copyright $\odot 2020$ Korean Society of Obstetrics and Gynecology 


\section{Obstetrics \& Gynecology Science}

Jeong Soo Cho, et al. Malakoplakia case mimicking EM cancer

\section{Case report}

A 52-year-old woman presented with intermittent left lower quadrant pain that had started in 2017. She was diagnosed with systemic lupus erythematosus 19 years ago and had been taking oral corticosteroids since then. Her past medical history also showed steroid-induced diabetes mellitus, hypertension, and hypothyroidism, which was treated with levothyroxine. Menopause occurred when she was in her thirties.

On her first visit to the emergency department in another tertiary hospital in May 2018, she underwent abdominal pelvic computerized tomography (APCT), which showed a multiloculated lesion on the left adnexa, with left ovary involvement and hydrosalpinx or pyosalpinx. Under the impression of a TOA, she was admitted and received conservative care including intravenous (IV) antibiotics for 15 days. However, the pain in her left lower quadrant persisted. She visited the emergency department for the second time in July 2018. APCT was performed again, which revealed a slightly increased multiloculated, rim-enhancing lesion compared with the previous APCT findings. Again, the impression was TOA; however, APCT showed a newly developed wall enhancement of the left ureter due to either secondary change or combined ureteritis. Because of persistent pain, she agreed to undergo surgery. On August 3, 2018, the patient underwent laparoscope-guided bilateral salpingo-oophorectomy. The pathology report showed chronic active inflammation with foam cell collection in her left ovary and both salpinges.

Because the surgery did not alleviate her pain, she underwent both APCT and magnetic resonance imaging in April 2019, which revealed a 7-cm irregular mass in her left adnexa and an infiltrative lesion in her uterus, suggestive of endometrial cancer. Imaging results also showed left ureter invasion by the left adnexal tumor. As prophylactic management, a left ureter stent was inserted. Furthermore, she underwent dilatation and curettage for diagnosis. Pathological examination showed chronic active inflammation with foam cell collection, consistent with the finding in the previous laparoscopic surgery.

The patient visited the emergency department of our hospital on May 20, 2019. She had sepsis and a body temperature of $>39^{\circ} \mathrm{C}$; blood culture revealed the presence of extended-spectrum beta-lactamase-producing Escherichia coli. The cervix culture taken at the emergency department also showed the presence of the same organism. Her initial blood urea nitrogen level was $45.6 \mathrm{mg} / \mathrm{dL}$, and her creatinine level was $2.75 \mathrm{mg} / \mathrm{dL}$, which implied septic postrenal acute kidney injury. For septic shock evaluation, she underwent APCT without contrast, which revealed newly developed mild hydronephrosis of the left kidney. The left adnexal mass remained the same as that seen in April 2019 (Fig. 1). We started initial shock management in the emergency department and conservative care including an IV antibiotic (meropenem) for bacteremia and left percutaneous nephrostomy (PCN) for left hydronephrosis and acute kidney injury.

After her recovery from septic shock, the patient underwent debulking surgery on June 4, 2019. During the surgery, severe adhesion in the pelvis involving the peritoneum, rectum, uterus, and left ureter was noted. Multiple hard and catheter-shaped foreign body-like materials were observed in the left parametrium and left pararectal space and near the left internal iliac artery. Caseous necrotic materials had spread deep into the left pelvic and paravaginal space. Total hysterectomy and debulking operation of the left pelvic mass, including left pelvic lymph nodes, were performed.

The final pathology showed foamy histiocyte aggregation
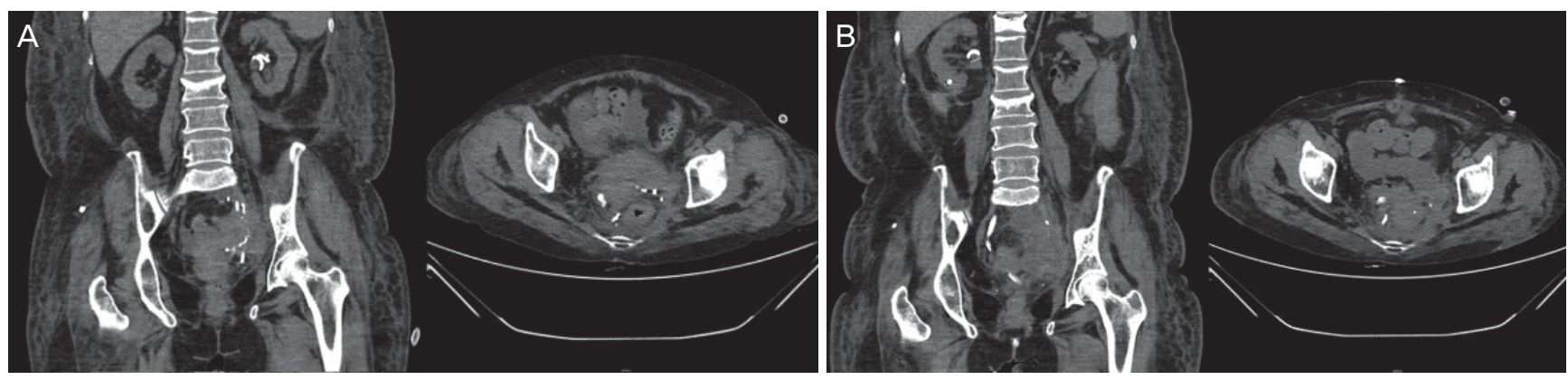

Fig. 1. (A) The patient's preoperative abdominal pelvic computerized tomography (APCT) depicting the pelvic mass (coronal view and horizontal view). (B) Postoperative day \#9 APCT depicting the recurred pelvic mass (coronal view and horizontal view). 


\title{
Obstetrics \& Gynecology Science
}

\author{
Vol. 63, No. 4, 2020
}

and acute and chronic inflammation with numerous Michaelis-Gutmann bodies, consistent with malakoplakia (Fig. 2). According to the recommended treatment for malakoplakia, we continued IV administration of tazoperan after the surgery. After the culture from peritoneal fluid cytology showed ceftazidime-sensitive $E$. coli, we changed the antibiotic to ceftazidime $1 \mathrm{~g}$ twice a day.

However, her creatinine level increased gradually to 4.48 $\mathrm{mg} / \mathrm{dL}$ after the surgery. A retrograde pyelogram showed patent left ureter and dye spillage from the right ureter. Right PCN was performed. A computerized tomography scan performed on postoperative day 9 showed recurrence of a 9-cm mass in her left pelvis. Hence, we again administered tazoperan. On postoperative day 21, the patient was referred to a general hospital for further conservative care with IV antibiotics.

On July 24, approximately 1.5 months after surgery, she underwent left PCN removal and right PCN clamping. On August 2, the patient returned to the emergency department with complaints of fever and right flank pain that had started on the previous day. She presented with right costovertebral angle tenderness and pyuria and was admitted to the urology department with a diagnosis of right acute pyelonephritis. On August 9, the anterograde pyelogram showed leakage at the right ureter, which was similar to the previous retrograde pyelogram finding. Right PCN exchange was performed. After conservative care with IV antibiotics, the patient was discharged on August 12. On September 10, direct ureteroneocystostomy was attempted; however, only right PCN exchange was performed because the ureter was short.

The patient has been closely followed up through outpatient visits to both urology and obstetrics and gynecology departments.

\section{Discussion}

Malakoplakia is a rare chronic inflammatory disease that usually affects the genitourinary tract [1], but it can affect any part of the body. However, its pathogenesis remains unknown. The disease usually occurs in immunosuppressed patients such as those with diabetes mellitus or those taking oral corticosteroids for rheumatic disease or immunosuppressants due to organ transplantation and autoimmune disease. Because of its characteristics, malakoplakia is assumed to be related to a defect in macrophage function against bacterial infection $[2,3]$.

In this case, malakoplakia resulted from bacterial infection in the pelvis that was first diagnosed as TOA and immunosuppression due to oral steroids and diabetes mellitus. Malakoplakia can affect various organs including the skin, lungs, and bones $[4,5]$. A literature review showed several reports of malakoplakia occurring in female pelvic organs involving the ovary and fallopian tube [6,7]; however, this is a very rare case of pelvic malakoplakia in the uterus without adnexa due to previous surgery and involving the full depth of the uterus, thus mimicking endometrial cancer as detected in imaging studies.

The diagnosis of malakoplakia is confirmed histologically after biopsy or surgery based on its pathognomonic features of von Hansemann histiocytes and Michaelis-Gutmann bodies, which are monocytes or macrophages containing undigested bacterial components including calcium [8].

The bladder is one of the organs most commonly affected with malakoplakia. Immunosuppressed patients such as those who underwent renal transplantation initially show hematuria or irritative symptoms [9]. However, malakoplakia affecting the female pelvis may not show disease-specific symptoms but rather general symptoms such as abdominal
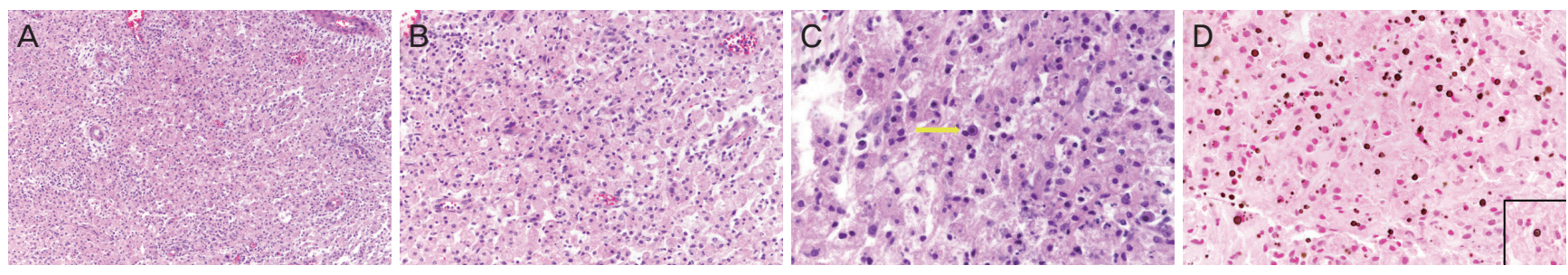

Fig. 2. (A, B) Foamy epithelioid histiocytes and some lymphocytes in endomyometrium and pelvic cavity (A: hematoxylin and eosin [H\&E] stain, $\times 100$, B: H\&E stain, $\times 200$ ). (C) Histiocytes with basophilic bodies containing calcium (H\&E stain, $\times 400)$ ). (D) Michaelis-Gutmann bodies noted (Von Kossa stain, $\times 400$ ). 


\section{Obstetrics \& Gynecology Science}

Jeong Soo Cho, et al. Malakoplakia case mimicking EM cancer

pain and mild fever. Without clinical suspicion in immunosuppressed patients, diagnosis can be delayed, thus increasing morbidity, as noted in this case.

The treatment of choice for malakoplakia is conservative care with IV antibiotics as soon as possible to prevent disease progression. Reduction of immunosuppression in addition to IV antibiotics should be considered if possible. The treatment period or regimen is not yet established. However, recent case studies have reported the use of IV broad-spectrum antibiotics for longer than 2 months [9-11]. If the lesion is localized to one organ, medical treatment is recommended; however, surgical resection followed by IV antibiotics should be considered if the disease forms a mass.

Because malakoplakia is a rare disease, physicians should have clinical suspicion for precise diagnosis. Without such awareness, examinations and imaging studies may lead to an incorrect diagnosis, as in this case. In this case, the patient showed only low abdominal pain, and she was initially diagnosed with TOA. Her pathologic report from the first surgery showed only chronic inflammation, and she was treated with IV antibiotics for only 2 weeks. The short treatment period allowed disease progression. Therefore, careful history review and physical examination are necessary. Malakoplakia presents as a hard and solid mass containing nodules, which is similar to malignant tumors in imaging studies. However, it can be differentiated by pathologic examination.

This report illustrates a case that was assumed to be endometrial cancer based on imaging studies but was diagnosed as pelvic malakoplakia after surgical resection. Because complications and morbidity increase with incorrect treatment, malakoplakia should be carefully diagnosed and treated. Malakoplakia should be suspected in immunosuppressed patients showing symptoms of infection, and differential diagnosis is therefore required.

\section{Conflict of interest}

No potential conflict of interest relevant to this article was reported.

\section{Ethical approval}

This study was approved by the Institutional Review Board of
Severance Hospital (4-2019-0988).

\section{Patient consent}

The Institutional Review Board approved the exemption of patients consent under the restriction that no identifiable personal information is revealed in the process.

\section{References}

1. Stanton MJ, Maxted W. Malacoplakia: a study of the literature and current concepts of pathogenesis, diagnosis and treatment. J Urol 1981;125:139-46.

2. Biggar WD, Crawford L, Cardella C, Bear RA, Gladman D, Reynolds WJ. Malakoplakia and immunosuppressive therapy. Reversal of clinical and leukocyte abnormalities after withdrawal of prednisone and azathioprine. Am J Pathol 1985;119:5-11.

3. Lewin KJ, Fair WR, Steigbigel RT, Winberg CD, Droller MJ. Clinical and laboratory studies into the pathogenesis of malacoplakia. J Clin Pathol 1976;29:354-63.

4. Bansal SS, Jokhi VH, Ponde SV, Kaushik N, Sonawane C. Malakoplakia of proximal tibia- A case report. J Orthop Case Rep 2014;4:78-80.

5. Dias PH, Slongo LE, Romero FR, Paques GR, Gomes RP, Rocha LC. Retroperitoneal sarcoma-like malakoplakia. Rev Assoc Med Bras (1992) 2011;57:615-6.

6. Chou SC, Wang JS, Tseng HH. Malacoplakia of the ovary, fallopian tube and uterus: a case associated with diabetes mellitus. Pathol Int 2002;52:789-93.

7. Aikat BK, Radhakrishnan VV, Rao MS. Malakoplakia--a report of two cases with review of the literature. Indian J Pathol Bacteriol 1973;16:64-70.

8. Lou TY, Teplitz C. Malakoplakia: pathogenesis and ultrastructural morphogenesis. A problem of altered macrophage (phagolysosomal) response. Hum Pathol 1974;5:191-207.

9. Hina S, Hasan A, Iqbal N, Shabbir MU, Sheikh AA. Malakoplakia of the urinary bladder and unilateral ureter. J Coll Physicians Surg Pak 2019;29:582-4.

10. Mitchell A, Dugas A. Malakoplakia of the colon following renal transplantation in a 73 year old woman: report of a case presenting as intestinal perforation. Diagn 


\section{Obstetrics \& Gynecology Science}

Vol. 63 , No. 4, 2020

Pathol 2019;14:22.

11. Dong H, Dawes S, Philip J, Chaudhri S, Subramonian
K. Malakoplakia of the urogenital tract. Urol Case Rep 2014;3:6-8. 